\title{
Two models of the influenza A M2 channel domain: verification by comparison Lucy R Forrest ${ }^{1}$, William F DeGrado², GR Dieckmann² and Mark SP Sansom ${ }^{1}$
}

Background: The influenza M2 protein is a simple membrane protein, containing a single transmembrane helix. It is representative of a very large family of single-transmembrane helix proteins. The functional protein is a tetramer, with the four transmembrane helices forming a proton-permeable channel across the bilayer. Two independently derived models of the M2 channel domain are compared, in order to assess the success of applying molecular modelling approaches to simple membrane proteins.

Results: The $\mathrm{C} \alpha \mathrm{RSMD}$ between the two models is $1.7 \AA$. Both models are composed of a left-handed bundle of helices, with the helices tilted roughly $15^{\circ}$ relative to the (presumed) bilayer normal. The two models have similar pore radius profiles, with a pore cavity lined by the Ser31 and Gly34 residues and a pore constriction formed by the ring of His37 residues.

Conclusions: Independent studies of M2 have converged on the same structural model for the channel domain. This model is in agreement with solid state NMR data. In particular, both model and NMR data indicate that the M2 helices are tilted relative to the bilayer normal and form a left-handed bundle.

Such convergence suggests that, at least for simple membrane proteins, restraints-directed modelling might yield plausible models worthy of further computational and experimental investigation.
Addresses: ${ }^{1}$ Laboratory of Molecular Biophysics, The Rex Richards Building, Department of Biochemistry, University of Oxford, South Parks Road, Oxford, OX1 3QU, UK. '2Department of Biochemistry and Biophysics, University of Pennsylvania School of Medicine, Philadelphia, PA 19104-6059, USA.

Correspondence: Mark SP Sansom

E-mail: mark@biop.ox.ac.uk

Key words: influenza virus, ion channel, membrane protein, molecular modelling, transbilayer pore

Received: 20 May 1998

Revisions requested: 23 June 1998

Revisions received: 08 September 1998

Accepted: 15 September 1998

Published: 05 November 1998

http://biomednet.com/elecref/1359027800300443

Folding \& Design 05 November 1998, 3:443-448

(C) Current Biology Ltd ISSN 1359-0278

\section{Introduction}

Ion channels are integral membrane proteins, enabling rapid $\left(\sim 10^{7}\right.$ ions $\left.\mathrm{s}^{-1}\right)$ passive movement of selected ions across biological membranes [1]. A number of viruses encode simple ion channel proteins, including M2 from influenza A [2-4], NB from influenza B [5,6] and $\mathrm{Vpu}$ from HIV-1 [7,8]. These viral ion channel proteins are of interest from a basic protein science perspective in that their relative simplicity (subunits of $\sim 100$ amino acids) makes them amenable to structural analysis. From a biomedical point of view, they are potential targets for antiviral drug therapy, and indeed $\mathrm{M} 2$ is the target of amantadine $[9,10]$, a drug effective against influenza $\mathrm{A}$.

M2 is an essential component of the influenza A viral envelope, forming proton-selective ion channels that are activated by the lowered $\mathrm{pH}$ to which the virus is exposed when in the endosome of an infected cell. M2 is a small protein (97 residues), containing a single transmembrane (TM) domain of $\sim 20$ residues close to its $\mathrm{N}$ terminus, which corresponds to the exterior of the virus particle. Channel-perturbing mutations map to the TM domain [11]. A synthetic peptide corresponding to this domain is $\alpha$-helical [12,13] and forms amantadine-sensitive channels when incorporated into lipid bilayers [14]. It is therefore thought that a bundle of TM helices form the transbilayer pore. The main form of the channel is a parallel bundle of four TM helices. SDS-PAGE and chemical cross-linking analyses demonstrate that the M2 protein is predominantly tetrameric. Furthermore, expression of mixed oligomers of wild-type and amantadine-resistant M2 in oocytes results in a fraction of amantadine-sensitive current consistent with a tetrameric channel [15-17]. A high-resolution structure of M2 has yet to be determined, although in principle it is amenable to analysis by, for example, solid state NMR methods [12]. Indeed, although secondary structure prediction analyses of several genomes have suggested that single-TM helix membrane proteins may predominate [18], few structural data are available for them. The relative simplicity of such proteins and of the oligomeric assemblies of their TM domains has recommended several of them (e.g. influenza A M2 [19,20], HIV-1 VPU [21], glycophorin A [22], and phospholamban [23]) to investigation by the systematic application of molecular modelling procedures. Although such computational approaches are well established and have been tested against functional data from, for example, channel-forming peptide antibiotics [24,25], the absence of hard structural data has made it difficult to evaluate their success. Here, we present a detailed comparison of two models of the M2 helix bundle $[19,20]$ which were generated independently using very different methods. It will be seen that these models are remarkably similar and in agreement with solid state NMR data [12]. 
This suggests that molecular modelling approaches can yield structural insights into these simple yet biologically important families of integral membrane proteins.

\section{Results}

\section{Two models of the M2 pore}

Our two research groups have taken different approaches to modelling the structure of the TM domain of M2. The procedure of Sansom et al. [19] was computationally intensive, using restrained molecular dynamics to generate structures that were then evaluated for consistency with experimental results. By contrast, DeGrado and colleagues [20] used an experimentally intensive approach in which a mathematical analysis of the results of site-directed mutagenesis was used to dictate the details of the packing geometry of the TM helices. The two models of the M2 TM helix bundle (henceforth the 'molecular dynamics' (MD) model [19] and the 'experimentally constrained' (EC) model [20]) were generated from the following sequences:

MD: $\quad$ L $^{26}-\mathrm{V}-\mathrm{I}-\mathrm{A}-\mathrm{A}-\mathrm{S}^{31}-\mathrm{I}-\mathrm{I}-\mathrm{G}^{34}$-I-L-H ${ }^{37}-\mathrm{F}-\mathrm{I}-\mathrm{L}-\mathrm{W}-\mathrm{I}-\mathrm{L}^{43}$

EC: $\quad$ L $^{26}-\mathrm{V}-\mathrm{V}-\mathrm{A}-\mathrm{A}-\mathrm{S}^{31}-\mathrm{I}-\mathrm{I}-\mathrm{G}^{34}-\mathrm{I}-\mathrm{L}-\mathrm{H}^{37}$-L-I-L-W-I-L-D ${ }^{44}$

Underlying both models was the assumption that the M2 helix bundle was made up of four approximately parallel TM helices, as indicated by a range of experimental data. In both models, all four His37 residues were in their neutral (i.e. deprotonated) form.

For the MD model, an initial idealised $\mathrm{C} \alpha$ template was generated in which each of the four helices was rotated about its long axis and tilted tangentially by $5^{\circ}$ so as to direct the C $\alpha$ atoms of residues 27, 30, 31, 34, 37, 38 and 41 towards the centre of the pore. These residues had been implicated in a number of studies as influencing M2 channel properties [11]. This $\mathrm{C} \alpha$ template was subsequently used in MD simulations (run with X-PLOR [26]) using a simulated annealing protocol plus distance restraints to maintain $\alpha$-helicity of the TM segments and to hold together adjacent helices of the bundle.

The EC model was derived from an analysis of the properties of a series of mutations to the TM region of M2. The degree to which a mutation perturbs the function of the channel varies with respect to the position in the sequence, reflecting the geometry of the structure of the bundle. Fourier analysis provides the phase and period of the distribution; the phase provides information concerning the rotation of the helices (about their helical axes) within a helix bundle, whereas the period can be related to the helix/helix packing angle. A series of models were generated that were each consistent with these parameters, and their geometries were optimised using only energy minimization. Thus, the MD approach used by Sansom $e t$ al. [19] explores a large amount of conformational space and relies on the molecular details of the TM segment to
Figure 1
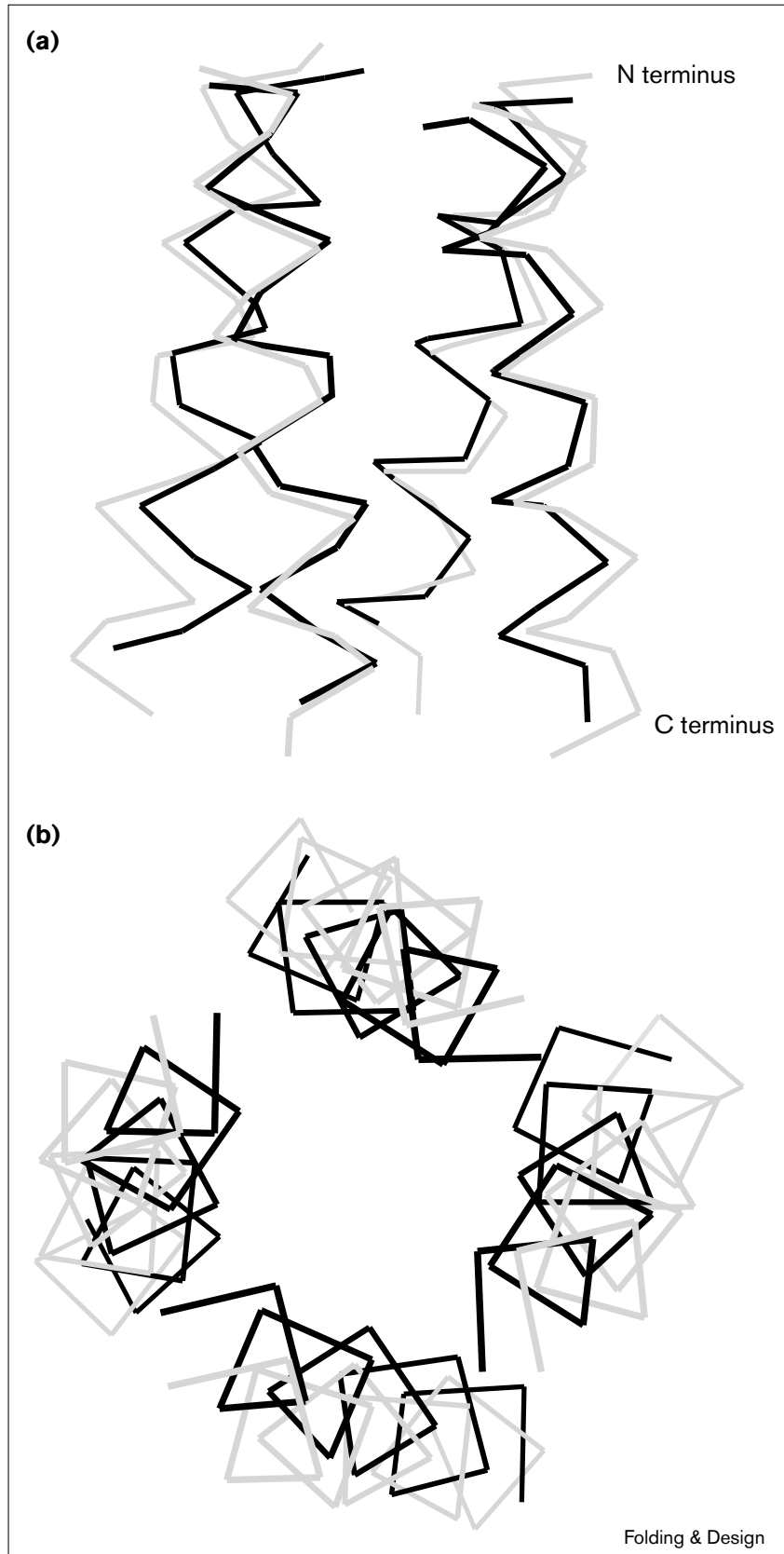

Superimposed $\mathrm{C} \alpha$ traces for the two models (EC, grey; MD, black). (a) View perpendicular to the pore axis, $\mathrm{N}$ termini at the top. (b) View down the pore axis, showing the left-handed supercoil.

dictate the structure of the model, whereas the EC models are much more constrained by the experimental data [20].

\section{Comparison of models}

For the current comparison, the structure with the greatest degree of fourfold rotational structure was selected from the ensemble of 25 structures generated by the MD procedure. And from the $29 \mathrm{EC}$ models, the structure closest 
Table 1

\begin{tabular}{lcccc}
\multicolumn{2}{l}{ Comparison of helix bundle geometries. } \\
\hline Model & $\begin{array}{c}\text { Helix tilt } \\
\text { angle }\left({ }^{\circ}\right)\end{array}$ & $\begin{array}{c}\text { Helix crossing } \\
\text { angle }\left(^{\circ}\right)\end{array}$ & \multicolumn{2}{c}{$\begin{array}{c}\text { Helix-helix } \\
\text { distance }(\AA)\end{array}$} \\
\cline { 3 - 5 } & & $\begin{array}{c}\text { N-terminal } \\
\text { half }\end{array}$ & $\begin{array}{c}\text { C-terminal } \\
\text { half }\end{array}$ \\
\hline EC & $15.2( \pm 0.2)$ & $+21( \pm 1)$ & 11.4 & 12.6 \\
MD & $15.6( \pm 0.5)$ & $+22( \pm 4)$ & $9.3( \pm 0.3)$ & $10.3( \pm 0.5)$ \\
\hline
\end{tabular}

For each helix, a vector was defined between the midpoint of the $\mathrm{C} \alpha$ atoms of its $\mathrm{N}$-terminal half (residues 26-32) and the midpoint of its $\mathrm{C}$-terminal half (residues 37-43). These vectors were used to calculate the quantities given in this table. Standard deviations refer to averages across all helices or all helix pairs within a model.

to the centroid was selected. The two models have been compared in terms of structural similarity per se, and in terms of the functional implications of their structures. A comparison of root mean square deviations (RMSDs) after superposition of the two structures yields a $C \alpha$ RMSD of $1.7 \AA$, and for all atoms (i.e. mainchain and sidechain) the RMSD is $2.5 \AA$. These figures should be compared with a C $\alpha$ RMSD of $1.1 \AA$ within the cluster of EC models and of $2.2 \AA$ within the ensemble of MD models. Thus, the difference between the two independently derived models is smaller than the variation between different versions of the same model.

Visual comparison of the two superimposed structures (Figure 1) reveals that in both models the helices form a left-handed supercoil and are packed more closely at their $\mathrm{N}$ termini than at their $\mathrm{C}$ termini. Comparison of their helix-packing geometry (Table 1) reveals that the helixcrossing angle is the same $\left(\sim+22^{\circ}\right)$ for both models, indicating the same left-handed supercoil structure. This results in a tilt angle of $\sim 15^{\circ}$ of the helix axes relative to the (presumed) bilayer normal. This may be compared with the solid state NMR results [12], which suggest that a synthetic M2 TM peptide incorporated into dimyristoyl phosphatidyl choline (DMPC) lipid bilayers is $\alpha$-helical and significantly tilted away from the bilayer normal. The tilt angle for the models $\left(15^{\circ}\right)$ is somewhat less than that indicated by the NMR data $\left(\sim 30^{\circ}\right)$, but, as discussed by Kovacs and Cross [12], there may be conformational or orientational heterogeneity in the preparation used for the solid state NMR and so it seems unlikely that the difference between these two tilt angles is significant. The EC and MD models do differ by $\sim 1 \AA$ in their interhelix distance, the helices being closer together in the MD than in the EC models. The origin of this difference is uncertain, but may reflect the different parameter sets and search methods used in the two procedures. RMSDs between the models as a function of residue number are examined in Figure 2. The C $\alpha$ RMSD is higher at the helix termini $(\sim 2 \AA)$ than in the middle of the TM helices $(\sim 1 \AA)$. Analysis of all-atom
Figure 2

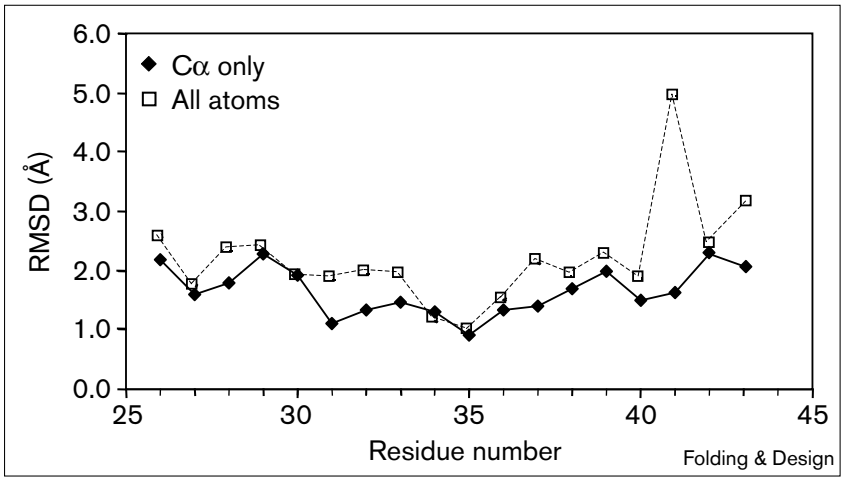

Comparison of EC and MD models. C $\alpha$ and all-atom RMSDs are plotted against residue number.

RMSDs reveals the greatest difference to be at $\operatorname{Trp} 41$, reflecting differences in sidechain conformation for this residue. In the vicinity of the functionally important His37 residue, the all-atom RMSD is only $\sim 2.3 \AA$.

\section{Functional implications}

In addition to comparison of the structures per se, one can compare the possible functional implications of the two models. Display of the molecular surface of the pore lining (Figure 3) reveals an N-terminal pocket or widening of the pore, lined in both models by residues Ser31 and Gly34. This has been suggested as a possible binding site for the channel-blocking drug amantadine [20], which has a van der Waals' diameter of $\sim 8 \AA$ and is approximately spherical in shape. Thus, some conformational change of either model would be required to accommodate this drug molecule. In particular, the pocket would have to become somewhat longer than in the current models (see below). Both models exhibit a constriction of the pore by the inward-pointing sidechains of His37. This histidine has been implicated in the mechanism of low $\mathrm{pH}$ activation of the channel, although alternative proposals have been made for the exact mechanism of such activation. Furthermore, the apparent affinity of amantadine for M2 increases as the $\mathrm{pH}$ is increased from 5.5 to about 7 (hence decreasing the protonation of the His residues). This suggests that the histidine residues may interact, either directly or indirectly, with the protonated amine group of amantadine.

The dimensions of the two pore models may be compared by comparison of pore radius profiles (Figure 4). There is a noticeable difference in their average (from $\mathrm{N}$ terminus to $\mathrm{C}$ terminus) pore radii ( 2.5 $\AA$ for $\mathrm{EC}$ and $\sim 1.3 \AA$ for $\mathrm{MD})$, reflecting the closer packing of the helices in the MD model. In both models, however, there is a constriction at the N-terminal mouth of the pore $(z=\sim-12 \AA$ followed by widening to give the pocket in the vicinity of Ser31 and Gly34 at $z=\sim-5 \AA$, and then a constriction in 
Figure 3

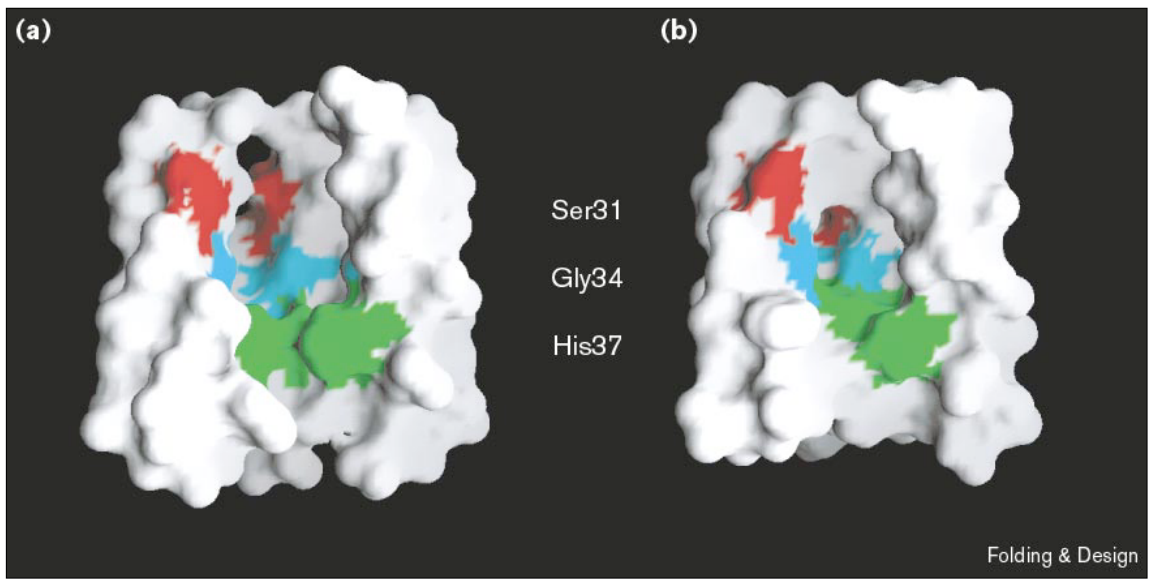

Molecular surfaces of the two models, drawn using GRASP [33]. (a) EC model. (b) MD model. Both structures are viewed perpendicular to the pore axis $(\mathrm{N}$ termini at the top), with the foremost helix removed to reveal the inside of the pore.

the region of His37 $(z=\sim+4 \AA)$. Despite these differences, the qualitative similarity in the shapes of the pore radius profiles of the two models is remarkable.

The widening of the pore near the centre of the bilayer (in the vicinity of Gly34) may be of functional significance. This is where one would expect the image charge associated with a permeating proton to be largest. By widening the pore in this region, it may be possible to minimise the unfavourable dehydration energy. Interestingly, in MD

\section{Figure 4}

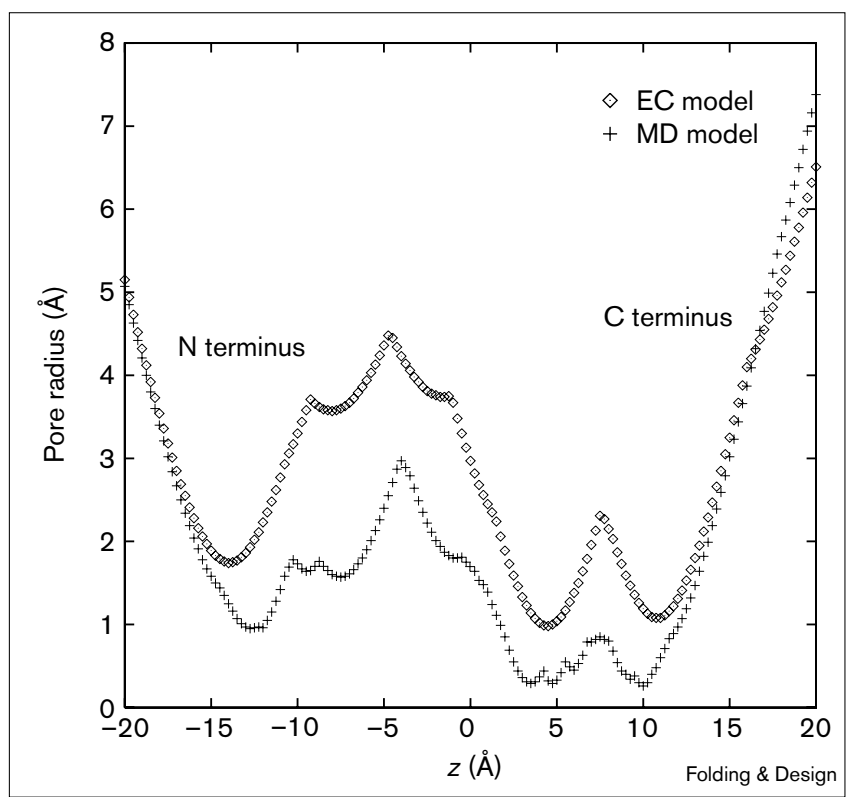

Pore radius profiles for the MD and EC models. For each model, the $\mathrm{N}$-terminal mouth of the pore is at $z=\sim-12 \AA$, the His37 ring is at $z=\sim+4 \AA$ and the C-terminal mouth is at $z=\sim+12 \AA$, where $z$ is the pore axis. simulations of an M2 helix bundle in a phospholipid bilayer, this pocket is associated with three water molecules throughout the duration ( $4 \mathrm{~ns}$ ) of the simulation $[27,28]$. Such waters could help to solvate an ion within the pocket. The existence of such a water-filled pocket within the centre of the M2 channel provides an intriguing parallel with the structure of a bacterial $\mathrm{K}^{+}$channel, KcsA [29]. KcsA also has a central water-filled cavity, although it is somewhat larger than that of M2, with a volume approximately equivalent to $\sim 10-15$ water molecules. Furthermore, recent spin-labelling experiments [30] suggest that the X-ray structure of KcsA, determined at neutral $\mathrm{pH}$, might correspond to the closed state of the channel, with an increase in pore radius at the intracellular mouth of the pore underlying channel activation. It will be intriguing to see whether a similar channel gating mechanism might apply for influenza M2, whereby an increase in the pore radius at, for example, the $\mathrm{N}$-terminal mouth of the helix bundle opens up the pocket, making it accessible to protons and to amantadine.

\section{Discussion}

To what extent are the two models independent? They are both based on mutation data, although this was more extensive for the EC than for the MD model. Thus, the EC model relied more on the experimental data to generate helix packings which were refined by energy minimisation, whereas the MD model used a more wide-ranging conformational search. Both models had similar starting assumptions, namely that the pore was formed by a parallel four-helix bundle and that the mutation data identified the pore-lining sidechains. The geometries of the computed structures did not depend on the interhelical tilt of the starting template. The EC models were generated using straight helices tilted by $14^{\circ}$ based on a Fourier analysis of the mutational data. Also, a set of starting models with parallel helices (i.e. tilted by $0^{\circ}$ ) was examined. Finally, coiled coils were evaluated as starting 
structures. Upon energy minimization, all three classes of structures converged to a single low-energy ensemble with an RMSD of $1.1 \AA$. Similarly, although the starting template for generation of the MD model had the TM helices tilted by $5^{\circ}$, previous studies [31] have shown that helix tilts can change markedly during helix bundle modelling by restrained MD. It seems unlikely, therefore, that a $5^{\circ}$ initial tilt biased the final model unduly. In a more general context, it might be argued that such indirect structural data and starting assumptions might be typical of a number of simple membrane proteins $[23,32]$.

In summary, these two models are remarkably similar and in broad agreement with solid state NMR data. The general significance of these results is the independent convergence on a single model for a simple TM helix bundle. This suggests that modelling studies may indeed play a valuable role in structure determination for the simplest (yet most populous) class of integral membrane proteins, especially given the relatively high accuracy of secondary structure and topology prediction for such proteins. The MD model has undergone refinement by prolonged (4 ns) unrestrained MD simulations in an explicit phospholipid bilayer [27,28]. Significantly, this does not seem to result in any great change in the helix bundle geometry from that reported here.

\section{Materials and methods}

The EC model was generated as described in [20]. Briefly, cysteine scanning mutagenesis of the TM helix of M2 was combined with electrophysiological assay of the effects of mutation on reversal potentials, ionic currents and amantadine resistance of currents. The periodicity of perturbation of channel properties as a function of the position of the mutation was analysed by a Fourier method. The results of this analysis were consistent with a left-handed interhelix crossing angle, and the parameters were used to generate both straight helix bundle and coiled coil starting models which, upon in vacuo energy minimisation, converged to a single set of structures with an average backbone RMSD of $1.1 \AA$. From this set of structures, the one closest to the average structure was used for the analyses in this paper.

The MD model was generated as described in [19] using a modification of the in vacuo restrained MD procedure described by [31]. Briefly, a $\mathrm{C} \alpha$ template formed the starting point, defining the initial positions of the $\mathrm{C} \alpha$ atoms of the M2 helices within an idealised parallel bundle, and thus embodying the assumptions discussed above. The main consequence of these assumptions was that the constituent helices of the bundle were rotated about their long axes such that their channel-lining faces (defined above) were directed towards the centre of the pore. Furthermore, the individual helices are tilted $5^{\circ}$ relative to the pore axis (z) such that the overall geometry of the bundle is that of a left-handed supercoil. Such tilting of the helices ensures that all of the residues identified by channel-perturbing mutations [12] (i.e. residues 27, 30 , $31,34,37,38$ and 41 ) were directed towards the lumen of the pore.

Having defined the $\mathrm{C} \alpha$ template, the remaining backbone and sidechain atoms were superimposed on the $\mathrm{C} \alpha$ atoms of the corresponding residues. The $\mathrm{C} \alpha$ atoms of the helices remained fixed throughout stage 1 of model generation. Annealing started at $1000 \mathrm{~K}$, during which weights for covalent terms were gradually increased and a repulsive van der Waals' potential was slowly introduced after an initial delay. Electrostatic terms were not included during stage 1. Stage 1 was repeated five times for each $\mathrm{C} \alpha$ template, each resultant structure being subjected to five restrained MD runs (stage 2), resulting in an ensemble of $5 \times 5=25$ final structures. During stage 2, distance restraints (both intrahelical and interhelical) were introduced, replacing the positional restraints on the positions of $\mathrm{C} \alpha$ atoms used in stage 1, and electrostatic interactions were gradually introduced into the potential energy function. From the ensemble of 25 structures thus generated, the structure with the highest rotational symmetry was selected for the analysis in this paper.

Models were displayed, superimposed and compared using QUANTA (Biosym/MSI). Pore surface diagrams were generated using GRASP [33]. Radius profiles were calculated using HOLE [34,35], which fits a largest possible sphere at successive points along the length of the pore.

\section{Acknowledgements}

Work in MSPS's lab is supported by the Wellcome Trust. LRF is an MRC student. Our thanks to Tim Cross and Frank Kovacs for discussions and for access to their solid state NMR data, and to Jim Lear, Larry Pinto, Robert Lamb, Igor Chizhmakov, Alan Hay and David Ogden for many helpful discussions concerning M2.

\section{References}

1. Hille, B. (1992). Ionic Channels of Excitable Membranes. (2nd edn) Sinauer Associates Inc., Sunderland, MA.

2. Pinto, L.H., Holsinger, L.J. \& Lamb, R.A. (1992). Influenza virus M2 protein has ion channel activity. Cell 69, 517-528.

3. Chizhmakov, I.V., Geraghty, F.M., Ogden, D.C., Hayhurst, A., Antoniou, M. \& Hay, A.J. (1996). Selective proton permeability and $\mathrm{pH}$ regulation of the influenza virus $\mathrm{M} 2$ channel expressed in mouse erythroleukaemia cells. J. Physiol. 494, 329-336.

4. Shimbo, K., Brassard, D.L., Lamb, R.A. \& Pinto, L.H. (1996). Selectivity and activation of the M2 ion-channel of influenza-virus. Biophys. J. 70, 1335-1346.

5. Betakova, T., Nermut, M.V. \& Hay, A.J. (1996). The NB protein is an integral component of the membrane of influenza-B virus. J. Gen. Virol. 77, 2689-2694.

6. Sunstrom, N.A., Prekumar, L.S., Prekumar, A., Ewart, G., Cox, G.B. \& Gage, P.W. (1996). Ion channels formed by NB, an influenza B virus protein. J. Memb. Biol. 150, 127-132.

7. Ewart, G.D., Sutherland, T., Gage, P.W. \& Cox, G.B. (1996). The Vpu protein of human immunodeficiency virus type 1 forms cation-selective ion channels. J. Virol. 70, 7108-7115.

8. Schubert, U., Ferrer-Montiel, A.V., Oblatt-Montal, M., Henklein, P., Strebel, K. \& Montal, M. (1996). Identification of an ion channel activity of the Vpu transmembrane domain and its involvement in the regulation of virus release from HIV-1-infected cells. FEBS Lett. $\mathbf{3 9 8}$, 12-18.

9. Hay, A.J., Wolstenholme, A.J., Skehel, J.J. \& Smith, M.H. (1985). The molecular basis of the specific anti-influenza action of amantadine. EMBO J. 4, 3021-3024.

10. Pinto, L.H. \& Lamb, R.A. (1995). Understanding the mechanism of action of the anti-influenza virus drug amantadine. Trends Microbiol. 3, 271.

11. Holsinger, L.J., Nichani, D., Pinto, L.H. \& Lamb, R.A. (1994). Influenza $A$ virus $\mathrm{M} 2$ ion channel protein: a structure-function analysis. J. Virol. 68, 1551-1563.

12. Kovacs, F.A. \& Cross, T.A. (1997). Transmembrane four-helix bundle of influenza A M2 protein channel: structural implications from helix tilt and orientation. Biophys. J. 73, 2511-2517.

13. Duff, K.C., Kelly, S.M., Price, N.C. \& Bradshaw, J.P. (1992). The secondary structure of influenza $\mathrm{A} M 2$ transmembrane domain. FEBS Lett. 311, 256-258.

14. Duff, K.C. \& Ashley, R.H. (1992). The transmembrane domain of influenza A M2 protein forms amantadine-sensitive proton channels in planar lipid bilayers. Virology 190, 485-489.

15. Holsinger, L.J. \& Lamb, R.A. (1991). Influenza virus M2 integral membrane protein is a homotetramer stabilized by formation of disulphide bonds. Virology 183, 32-43.

16. Sugrue, R.J. \& Hay, A.J. (1991). Structural characteristics of the M2 protein of influenza A viruses: evidence that it forms a tetrameric channel. Virology 180, 617-624.

17. Sakaguchi, T., Tu, Q.A., Pinto, L.H. \& Lamb, R.A. (1997). The active oligomeric state of the minimalistic influenza virus M2 ion channel is a tetramer. Proc. Natl Acad. Sci. USA 94, 5000-5005. 
18. Arkin, I.T., Brünger, A.T. \& Engelman, D.M. (1997). Are there dominant membrane protein families with a given number of helices? Proteins 28, 465-466.

19. Sansom, M.S.P., Kerr, I.D., Smith, G.R. \& Son, H.S. (1997). The influenza $A$ virus $M 2$ channel: a molecular modelling and simulation study. Virology 233, 163-173.

20. Pinto, L.H., et al., \& DeGrado, W.F. (1997). A functionally defined model for the M2 proton channel of influenza A virus suggest a mechanism for its ion selectivity. Proc. Natl Acad. Sci. USA 94, 11301-11306.

21. Grice, A., Kerr, I.D. \& Sansom, M.S.P. (1997). Ion channels formed by HIV-1 Vpu: a modelling and simulation study. FEBS Lett. 405 , 299-304.

22. Lemmon, M.A., Treutlein, H.R., Adams, P.D., Brünger, A.T. \& Engelman, D.M. (1994). A dimerisation motif for transmembrane $\alpha$ helices. Nat. Struct. Biol. 1, 157-163.

23. Adams, P.D., Arkin, I.T., Engelman, D.M. \& Brünger, A.T. (1995). Computational searching and mutagenesis suggest a structure for the pentameric transmembrane domain of phospholamban. Nat. Struct. Biol. 2, 154-162.

24. Breed, J., Biggin, P.C., Kerr, I.D., Smart, O.S. \& Sansom, M.S.P. (1997). Alamethicin channels - molecular modelling via restrained molecular dynamics simulations. Biochim. Biophys. Acta 1325, 235-249.

25. Breed, J., Kerr, I.D., Molle, G., Duclohier, H. \& Sansom, M.S.P. (1997) lon channel stability and hydrogen bonding. Molecular modelling of channels formed by synthetic alamethicin derivatives. Biochim. Biophys. Acta 1330, 103-109.

26. Brünger, A.T. (1992). X-PLOR Version 3.1. A System for $X$-ray Crystallography and NMR. Yale University Press, New Haven, CT.

27. Forrest, L.R. \& Sansom, M.S.P. (1998). Simulations of the M2 channel for influenza A virus. Biochem. Soc. Trans. 26, S303.

28. Sansom, M.S.P., Tieleman, D.P., Forrest, L.R. \& Berendsen, H.J.C. (1998). Molecular dynamics simulations of membranes with embedded proteins and peptides: porin, alamethicin and influenza M2. Biochem. Soc. Trans. 26, 438-443.

29. Doyle, D.A., Cabral, J.M., Pfuetzner, R.A., Kuo, A., Gulbis, J.M., Cohen, S.L., Cahit, B.T. \& MacKinnon, R. (1998). The structure of the potassium channel: molecular basis of $\mathrm{K}^{+}$conduction and selectivity. Science 280, 69-77.

30. Perozo, E., Cortes, D.M. \& Cuello, L.G. (1998). Three-dimensional architecture and gating mechanism of $\mathrm{a} \mathrm{K}^{+}$channel studied by EPR spectroscopy. Nat. Struct. Biol. 5, 459-469.

31. Kerr, I.D., Sankararamakrishnan, R., Smart, O.S. \& Sansom, M.S.P. (1994). Parallel helix bundles and ion channels: molecular modelling via simulated annealing and restrained molecular dynamics. Biophys. J. 67, 1501-1515.

32. Treutlein, H.R., Lemmon, M.A., Engelman, D.M. \& Brünger, A.T. (1992). The glycophorin A transmembrane domain dimer: sequence specific propensity for a right handed supercoil of helices. Biochemistry 31, 12726-12733.

33. Nicholls, A. \& Honig, B. (1992). GRASP Manual. Columbia University, New York.

34. Smart, O.S., Goodfellow, J.M. \& Wallace, B.A. (1993). The pore dimensions of gramicidin A. Biophys. J. 65, 2455-2460.

35. Smart, O.S., Breed, J., Smith, G.R. \& Sansom, M.S.P. (1997). A novel method for structure-based prediction of ion channel conductance properties. Biophys. J. 72, 1109-1126. 